\title{
Video Article \\ Encapsulation of Cardiomyocytes in a Fibrin Hydrogel for Cardiac Tissue Engineering
}

\author{
Kathy Yuan Ye ${ }^{1}$, Kelly Elizabeth Sullivan ${ }^{1}$, Lauren Deems Black ${ }^{1}$ \\ ${ }^{1}$ Department of Biomedical Engineering, Tufts University \\ Correspondence to: Lauren Deems Black at lauren.black@tufts.edu
}

URL: https://www.jove.com/video/3251

DOI: doi:10.3791/3251

Keywords: Bioengineering, Issue 55, fibrin, scaffold, hydrogel, cardiac tissue engineering, contraction force, neonatal cardiomyocytes

Date Published: 9/19/2011

Citation: Ye, K.Y., Sullivan, K.E., Black, L.D. Encapsulation of Cardiomyocytes in a Fibrin Hydrogel for Cardiac Tissue Engineering. J. Vis. Exp. (55), e3251, doi:10.3791/3251 (2011).

\section{Abstract}

Culturing cells in a three dimensional hydrogel environment is an important technique for developing constructs for tissue engineering as well as studying cellular responses under various culture conditions in vitro. The three dimensional environment more closely mimics what the cells observe in vivo due to the application of mechanical and chemical stimuli in all dimensions ${ }^{1}$. Three-dimensional hydrogels can either be made from synthetic polymers such as PEG-DA ${ }^{2}$ and PLGA ${ }^{3}$ or a number of naturally occurring proteins such as collagen ${ }^{4}$, hyaluronic acid ${ }^{5}$ or fibrin 6,7 . Hydrogels created from fibrin, a naturally occurring blood clotting protein, can polymerize to form a mesh that is part of the body's natural wound healing processes ${ }^{8}$. Fibrin is cell-degradable and potentially autologous ${ }^{9}$, making it an ideal temporary scaffold for tissue engineering

\begin{abstract}
Here we describe in detail the isolation of neonatal cardiomyocytes from three day old rat pups and the preparation of the cells for encapsulation in fibrin hydrogel constructs for tissue engineering. Neonatal myocytes are a common cell source used for in vitro studies in cardiac tissue formation and engineering ${ }^{4}$. Fibrin gel is created by mixing fibrinogen with the enzyme thrombin. Thrombin cleaves fibrinopeptides FpA and FpB from fibrinogen, revealing binding sites that interact with other monomers ${ }^{10}$. These interactions cause the monomers to self-assemble into fibers that form the hydrogel mesh. Because the timing of this enzymatic reaction can be adjusted by altering the ratio of thrombin to fibrinogen, or the ratio of calcium to thrombin, one can injection mold constructs with a number of different geometries ${ }^{11,12}$. Further we can generate alignment of the resulting tissue by how we constrain the gel during culture ${ }^{13}$.

After culturing the engineered cardiac tissue constructs for two weeks under static conditions, the cardiac cells have begun to remodel the construct and can generate a contraction force under electrical pacing conditions ${ }^{6}$. As part of this protocol, we also describe methods for analyzing the tissue engineered myocardium after the culture period including functional analysis of the active force generated by the cardiac muscle construct upon electrical stimulation, as well as methods for determining final cell viability (Live-Dead assay) and immunohistological staining to examine the expression and morphology of typical proteins important for contraction (Myosin Heavy Chain or MHC) and cellular coupling (Connexin 43 or Cx43) between myocytes.
\end{abstract}

\section{Video Link}

The video component of this article can be found at https://www.jove.com/video/3251/

\section{Protocol}

\section{Neonatal cardiomyocyte isolation - preparation (day before)}

Solutions created in this section: PBS-Glucose solution, stop media.

1. Prepare a PBS-glucose solution by adding $5 \mathrm{~mL}$ penicillin-streptomycin ( 100 units $/ \mathrm{ml}$ and $100 \mu \mathrm{g} / \mathrm{ml}$ respectively) and $1.98 \mathrm{~g}$ of glucose to $250 \mathrm{ml} 1 \mathrm{x}$ sterile phosphate buffered saline (PBS) and bring solution volume to $500 \mathrm{ml}$ with additional sterile 1x PBS.

2. Prepare stop media by adding $25 \mathrm{ml} \mathrm{FBS}$ and $5 \mathrm{ml}$ of penicillin-streptomycin (same concentration as above) to $250 \mathrm{ml}$ sterile Dulbecco's Modified Eagle's Medium (DMEM) and bring the volume to $500 \mathrm{ml}$ with sterile DMEM before sterile filtering through a 0.2 micron filter.

3. Sterilize surgical instruments needed for isolation by autoclaving: a hemostat, \#5 forceps, large scissors, micro-scissors, and a scalpel handle $(\# 4)$.

\section{Neonatal cardiomyocyte isolation - preparation (day of harvest)}

Be sure to maintain sterility

Solutions used in this section: PBS-glucose solution, Betadine 
1. For each litter, take the two sterile $100 \mathrm{~mm}$ petri dishes, place them in the hood and fill with $10 \mathrm{~mL}$ of ice-cold PBS-glucose. These should then be placed in an ice bucket filled with ice in the sterile culture hood.

2. Place a $250 \mathrm{~mL}$ beaker with $30-40 \mathrm{~mL}$ of Betadine into the hood.

3. Add $50 \mathrm{~mL} /$ litter of PBS-glucose into a bottle, seal and place into a $37^{\circ} \mathrm{C}$ water bath

4. For each person, place an absorbent bench underpad on the hood work surface and place a sterile drape on top being careful not to touch the center work area of the sterile drape. Dump the surgical instruments and a $4 \times 4$ gauze pad onto the sterile drape without touching the instruments. Open a sterile \#20 scalpel blade and dump onto drape, again being careful not to touch with non-sterile gloves.

5. Take the pups from the dam and place into opaque container, place pups into the hood

6. Put on sterile gloves

7. Fold the gauze into fourths, clamp with hemostat and place into Betadine beaker.

8. Put the scalpel blade onto the scalpel handle and set aside.

\title{
3. Neonatal cardiomyocyte isolation - heart dissection
}

\author{
Solutions used in this section: Betadine, PBS-glucose solution
}

1. Pick up the pup in your non- dominant hand by pinching skin between shoulder blades between the thumb and index finger. Using the large scissors, decapitate the pup in one cut. Be sure to cut from the back of the pup forwards, to ensure that the spine is completely severed.

2. Swab the pup's chest with the betadine soaked gauze. Secure the pup by pinching the shoulder blades together. Perform partial thoracotomy to expose the heart. Increase applied pressure, thereby forcing the heart past the ribs for scalpular dissection.

3. Run the scalpel blade behind the heart to sever the great vessels and remove the heart. Place the heart in the petri dish containing PBSglucose that is on ice.

4. Repeat steps 1-3 for each pup in the litter.

\section{Neonatal cardiomyocyte isolation - myocyte isolation}

\author{
Solutions created/used in this section: PBS-glucose solution, collagenase solution, stop solution
}

1. Once the hearts have been isolated, remove any residual blood and connective tissue by rinsing in ice-cold PBS glucose solution, remove the top $1 / 3$ of the heart to isolate only the ventricular tissue and place into a fresh petri dish with ice-cold PBS glucose, prepared earlier.

2. Carefully mince the hearts into 1 cubic $\mathrm{mm}$ using the micro-scissors and the forceps.

3. Take a sterile transfer pipette, cut off the tip using scissors so that the mouth of the pipette is $\sim 3 \mathrm{~mm}$ in diameter. Use the pipette to transfer the tissue pieces and all of the solution into a $50 \mathrm{ml}$ conical and place on ice.

4. Weigh out 15,000 units per litter of pups of type II collagenase (units $/ \mathrm{mg}$ is dependent on the lot) and place into the bottle of $37^{\circ} \mathrm{C}$ warmed PBS-glucose prepared previously to create collagenase solution. Mix well and sterile filter into a separate bottle. Place back into the $37^{\circ} \mathrm{C}$ water bath. Place the stop solution into the $37^{\circ} \mathrm{C}$ water bath as well.

5. Allow the minced tissue to settle to the bottom of the centrifuge tube. Remove the supernatant until the total volume is $\sim 10 \mathrm{~mL}$. Add $7 \mathrm{ml}$ of collagenase solution to the centrifuge tube.

6. Put the conical tube with the tissue pieces and the collagenase into a tube rack on an orbital shaker inside a $37^{\circ} \mathrm{C}$ incubator or oven. Turn the orbital shaker on at approximately $60 \mathrm{rpm}$ and close the door. Set a timer for 7 minutes. Be sure to place the collagenase back into the water bath to keep it warm.

7. When the timer goes off, bring the conical back into the hood. Also bring the warm collagenase and stop solution into the hood. Gently titrate the tissue pieces 5-7 times to break them up. After titration, allow the pieces to settle to the bottom (2-3 minutes). Aspirate off as much of the supernatant as possible being very careful not to suck up the tissue pieces. Afterwards, add $7 \mathrm{ml}$ of collagenase solution to the tissue pieces and place back into the incubator on the shaker for 7 minutes.

8. For each remaining step, gently titrate 10 times to break up the tissue pieces. Once the tissue pieces settle, draw the supernatant off and collect it in a separate $50 \mathrm{ml}$ conical. Add $7 \mathrm{ml}$ of collagenase to the tissue pieces and digest again for 7 minutes. To the supernatant tube, add $10 \mathrm{~mL}$ of stop solution with a different serological pipette after each addition of supernatant from the digestion.

9. Repeat until all of the collagenase has been used (7 steps in total).

10. After the final digestion step, take the conical with the cell solution and filter through $70 \mu \mathrm{m}$ cell sieve into fresh conical.

11. Spin cells down at $100 \mathrm{~g}$ for 5 minutes and resuspend in $20 \mathrm{~mL}$ of DMEM to be counted using a hemocytometer, and place the cells on ice.

12. Place $50 \mu \mathrm{l}$ of the cells into a Trypan Blue solution ( $75 \mu \mathrm{l}$ Trypan Blue, $125 \mu \mathrm{l}$ PBS), mix well before placing $10 \mu \mathrm{l}$ in the hemocytometer for counting. Live cells are clear while dead cells are blue. Expect approximately 3 million cells per rat pup, with a viability of approximately $80-90 \%$.

\section{Casting fibrin gel constructs - preparation for creating fibrin gels (done well in advance)}

Solutions created in this section: fibrinogen stock solution, thrombin stock solution, Pluronics solution, myocardial construct media.

1. Prepare a $33 \mathrm{mg} / \mathrm{mL}$ stock solution of fibrinogen in $20 \mathrm{mM}$ HEPES buffer in $0.9 \%$ saline by slowly mixing fibrinogen into the HEPES buffered saline over several hours at $37^{\circ} \mathrm{C}$. Allow the solution to settle overnight at $2-8^{\circ} \mathrm{C}$. Warm the solution to $37^{\circ} \mathrm{C}$. The solution is sterile filtered through a series of consecutive filters: $40 \mu \mathrm{m}$ cell strainers, $0.45 \mu \mathrm{m}$ bottle top filters with glass pre-filters, and $0.2 \mu \mathrm{m}$ bottle top filters with glass pre-filters. The solution is aliquoted into $1 \mathrm{~mL}$ and $3 \mathrm{~mL}$ aliquots and stored at $-20^{\circ} \mathrm{C}$.

2. Prepare a $25 \mathrm{U} / \mathrm{mL}$ stock solution of thrombin by adding $500 \mathrm{U}$ of thrombin to $18 \mathrm{~mL}$ of $0.9 \%$ saline and $2 \mathrm{~mL}$ of sterile deionized water, sterile filter through a $0.2 \mu \mathrm{m}$ filter, aliquot into $500 \mu \mathrm{l}$ and $250 \mu \mathrm{l}$ aliquots and freeze at $-80^{\circ} \mathrm{C}$.

3. Prepare a $5 \% \mathrm{w} / \mathrm{v}$ Pluronics F-127 solution, by dissolving $50 \mathrm{~g}$ of Pluronics F-127 to $700 \mathrm{~mL}$ of deionized water. Bring solution volume up to $1 \mathrm{~L}$ with additional deionized water. Sterile filter with $0.2 \mu \mathrm{m}$ filter. The Pluronics solution can be used up to three times before replacing if sterile filtered after each use. 
4. Prepare myocardial construct media by adding $10 \%$ horse serum, $2 \%$ fetal bovine serum, $1 \%$ penicillin-streptomycin, and $6 \mathrm{mg} / \mathrm{mL}$ aminocaproic acid into DMEM. $50 \mu \mathrm{g} / \mathrm{ml}$ of ascorbic acid and $2 \mu \mathrm{g} / \mathrm{ml}$ of insulin in $25 \mu \mathrm{M}$ HEPES need to be added immediately before feeding.

5. Assemble mandrel by putting together one teflon rod, one teflon sleeve, two teflon washers with a notch removed for injection purposes, and two rubber O-rings (see Figure 2a). Autoclave before use.

6. Take $6 \mathrm{cc}$ syringe casings for the outer part of the mold and 3cc syringe casings to be used as plungers, and prepare them by cutting off the luer -lock ends and autoclaving (see Figure 2a).

\title{
6. Casting fibrin gel constructs - preparation for creating fibrin gels (right before making the fibrin gel constructs)
}

Solutions used in this section: Pluronics solution

1. Sterile filter the $5 \%$ Pluronics F-127 solution with a $0.2 \mu \mathrm{m}$ filter before use. Place mandrels and syringe casings into $5 \%$ Pluronics solution in a $1 \mathrm{~L}$ beaker in the hood. Leave the parts soaking in the Pluronics solution for 2-3 hours in the hood to ensure complete coating. The Pluronics solution coats the mandrels and prevents the fibrin gel from adhering to the mandrels.

2. After the 2-3 hour incubation, pour the $5 \%$ Pluronics solution back into the bottle, place sterile drapes down on the surface of the hood and wear sterile gloves to construct the molds.

3. Place the constructed mandrels into the $6 \mathrm{cc}$ syringe casings, using the 3cc syringe as a plunger to ensure a tight seal between the o-rings and Teflon washers.

\section{Casting fibrin gel constructs via injection molding}

\author{
Solutions created in this section: F solution, $T$ solution, cell solution.
}

1. To make $1 \mathrm{~mL}$ of fibrin gel ( $3.3 \mathrm{mg} / \mathrm{mL}$ final fibrinogen concentration, $25 \mathrm{U} / \mathrm{mL}$ final thrombin concentration), create $\mathrm{F}$ solution in a conical tube, by adding $112 \mu \mathrm{l}$ of the fibrinogen stock to $558 \mu \mathrm{l}$ of $20 \mathrm{mM}$ HEPES Buffer in $0.9 \%$ saline solution. In a separate conical tube, create a $\mathrm{T}$ solution by adding $17 \mu \mathrm{l}$ of the thrombin stock, and $1.3 \mu \mathrm{l}$ of $2 \mathrm{~N} \mathrm{Ca++}$ solution to $135 \mu \mathrm{l}$ of DMEM. See Table 1.

2. In a third conical tube, prepare a cell solution by spinning down the cells and resuspending the cells in a volume so that the concentration of cell is 29.4 million cells $/ \mathrm{mL}$ or 6 times the concentration of the desired final concentration of cells in the construct

3. When you are ready to cast the fibrin gel construct, prep a $1 \mathrm{~mL}$ syringe with a $18 \mathrm{G} 1 \frac{1 / 2}{\mathrm{inch}}$ long needle. Have a $21 \mathrm{G} 1 \mathrm{inch}$ needle ready as well.

4. The fibrin solution is created at a 4:1:1 ratio of $F$ solution: $T$ solution: cell solution. To make one $\mathrm{mL}$ of gel, add $667 \mu \mathrm{L}$ of $\mathrm{F}$ solution into a clean $50 \mathrm{~mL}$ centrifuge tube, followed by $167 \mu \mathrm{L}$ of cell solution, and lastly add $167 \mu \mathrm{l}$ of T solution. Pipette to mix solution together being careful not to introduce bubbles. Once solutions are mixed, the reaction has started and the injection of the constructs should be done immediately.

5. Take the previously prepared syringe with the $18 \mathrm{G}$ needle and draw up fibrin solution. Take care not to invert the syringe to prevent bubbles from getting into the needle. Replace $18 \mathrm{G}$ needle with a $21 \mathrm{G}$ needle. Tap syringe gently to force out air bubbles.

6. Insert syringe into the mold between the stopper and casing following the groove in the Teflon O-ring and inject the solution into the mold. Tilt the mold with the groove on top to insure complete filling. Remove the syringe and continue to fill remaining molds. Enough gel solution can be created to fill several molds at the same time. However, because the solution gels quickly, it is generally a good idea to limit the number of constructs injected at a given time to 6 .

7. Wrap the molds in Parafilm in groups of three and place in the incubator or oven at $37^{\circ} \mathrm{C}$. Allow the gels to incubate in the molds for 20 minutes to allow the gel time to polymerize.

8. Fill each culture jar (Nalgene straight-side jar) with $21 \mathrm{~mL}$ of myocardial construct medium per construct. Use the sterile $3 \mathrm{cc}$ syringe casing as a plunger to force the mandrel with the construct into a large petri dish with DMEM. Then place the construct into the sample jar. Each 16 oz jar can hold up to 6 constructs, while each 4 oz jar can hold 2.

9. Screw the caps on the jars and transfer them to the incubator. Inside the incubator, loosen the caps on the jars to allow for gas exchange.

10. After 24 hours, take a sterile dental pick and push the construct away from the white teflon O-rings on the ends of the ring mold to ensure uniform alignment of the construct (See Figure 2 in representative results).

\section{Analysis techniques (after 2 weeks in culture) - contraction force testing}

\section{Solutions used in this section: DMEM, myocardial construct media.}

1. Clamp the alligator clips on the lead coming from the stimulator to the wires on the electrodes in the bath. Power up the data acquisition board, pulse generator, and force transducer. The force transducer should be switched to the $5 \mathrm{~g}$ setting and zeroed. Open a custom LabView program that displays and saves the data from the force transducer. Create a new, empty text file in the data folder for each sample.

2. Place DMEM into the $37^{\circ} \mathrm{C}$ water bath and allow time for it to warm up before testing the constructs. Once warmed, place $37^{\circ} \mathrm{C}$ DMEM into the medium bath of the force measurement system (see Figure $3 \mathrm{~A}$ ).

3. Remove the construct from the sample jar by gently sliding the construct ring from Teflon support with tweezers and place the construct over the fixed metal post in the force measurement system medium bath. Do not grip the construct with the tweezers! Rather, use the tweezers to push and lift the construct off of the mandrel support.

4. Place the other end of the construct over the transducer arm and tighten until the transducer reads $0.50 \mathrm{~V}$, (approximately 1.0 grams force or 10 millinewtons of tension)

5. Select the text file for the contraction force data to be recorded into.

6. On the cardiac stimulator (Model \# S88X, Grass Technologies), set the pulse voltage to $20 \mathrm{~V}(8 \mathrm{~V} / \mathrm{cm}), 6 \mathrm{~ms}$ duration, and a rate of $1 \mathrm{~Hz}$.

7. Start the electrical pacing by pressing the "output on/off" button 
8. Start recording until waveform becomes regular.

9. Carefully remove the construct from the force measurement system medium bath and place it back into culture medium. Then remove the DMEM from the force measurement system medium bath and replace with fresh, warm DMEM to analyze additional samples.

10. Cut the construct and unroll it so that you can measure the length and width of the construct

11. Cut the construct into sections to be used for additional analysis measurements including viability, histology, or western blot measurements.

\section{Analysis techniques (after 2 weeks in culture) - Live-Dead Assay for viability (with Invitrogen Live/Dead Assay) ${ }^{14}$ :}

Solutions used in this section: EthD-1 stock solution, calcein AM stock solution PBS

1. Rinse samples with $3 \times 5$ minute washes in PBS

2. Add $20 \mu \mathrm{l}$ of $2 \mathrm{mM}$ EthD-1 stock solution to $10 \mathrm{~mL}$ of sterile PBS and vortex to ensure thorough mixing.

3. Add $5 \mu \mathrm{l}$ of $4 \mathrm{mM}$ calcein AM stock stolution to EthD-1 solution. Again, vortex to ensure thorough mixing.

4. Add enough volume of the above solution to cover the construct.

5. Incubate covered (to prevent photobleaching of the dyes) for 30 minutes at room temperature.

6. Remove the dyes and replace with warm PBS. Observe and record images with a fluorescent microscope. Calcein is excited by $494 \mathrm{~nm}$ light and emits $517 \mathrm{~nm}$ light, while ethidium homodimer-1 is by $528 \mathrm{~nm}$ light and emits $617 \mathrm{~nm}$ light. See Figure 4 for sample results.

\section{Analysis techniques (after 2 weeks in culture) - immunohistochemistry for important myocyte proteins:}

Solutions used in this section: PBS, 4\% paraformadehyde in PBS, 5\% donkey serum in PBS, antibodies in PBS, $0.1 \mathrm{ng} / \mathrm{mL}$ Hoechst 33258 in PBS.

1. Rinse samples with $3 \times 5$ minute washes in PBS.

2. Fix with $4 \%$ paraformaldehyde in PBS solution, for $2-3$ hours at $4{ }^{\circ} \mathrm{C}$

3. Rinse samples with $3 \times 5$ minute washes in PBS.

4. The sample can now be embedded, sectioned and stained according to the protocol of choice. The remaining portion of this protocol covers whole construct staining for imaging with confocal microscopy. Note that the incubation times are longer than that for sectioned tissue as there needs to be more time for the antibodies to diffuse into the construct. In addition, all of the remaining steps are conducted at room temperature.

5. Add $0.1 \%$ Triton-X in PBS to the samples for 30 minutes to permeabilize the cell membranes.

6. Rinse the samples with $3 \times 10$ minute washes in PBS.

7. Add $5 \%$ donkey serum in PBS to the samples for $1 \frac{1}{2}$ hours to block any non-specific binding of the secondary antibody to the samples.

8. Add Connexin 43 (1:50 dilution) and Myosin Heavy Chain (1:100 dilution) primary antibodies in PBS to the samples for 3 hours. In order to label both proteins consecutively you must make sure that the primary antibodies are from different hosts (i.e. rabbit and mouse).

9. Rinse the samples with $3 \times 10$ minute washes in PBS.

10. Add the appropriate fluorescently labeled secondary antibodies in PBS for 3 hours.

11. Rinse the samples with $3 \times 10$ minute washes in PBS

12. Just before imaging the samples on the confocal microscope, add $0.1 \mathrm{ng} / \mathrm{ml} \mathrm{Hoechst} 33258$ in PBS for 15 minutes.

13. Rinse the samples with $3 \times 10$ minute washes in PBS.

14. Analyze the samples expression of MHC and Cx43 by imaging the cells with a confocal microscope (See Figure 4 for example result).

\section{Representative results / Outcomes:}

The cardiomyocyte fibrin construct initially covers the entire width of the mold (Figure 2B). No bubbles should exist in the construct and it should look uniform across the entire length. After two weeks of culturing, the construct contract to approximately $1 / 4$ of the initial width (Figure 2C).

When the construct is electrically paced in our custom contraction force device (Figure 3A), twitch force data can be generated as shown in Figure 3B. The waveform can be analyzed separately in MATLAB (MathWorks) to determine the force, rate of contraction, and rate of relaxation. Twitch forces of approximately $1.3 \mathrm{mN}$ are expected ${ }^{6}$

Cell viability of the construct is dependent on the depth of the construct, due to the diffusion limitations of oxygen into the construct. On the surface of the construct, Figure 4A, high cell viability is observed. With confocal microscopy, Figure 4B, the aligned structure of the construct is observed due to the Myosin Heavy Chain, MHC, is important for contraction, shown in red. Connexin 43, shown in green, is necessary for cellular coupling between myocytes. 


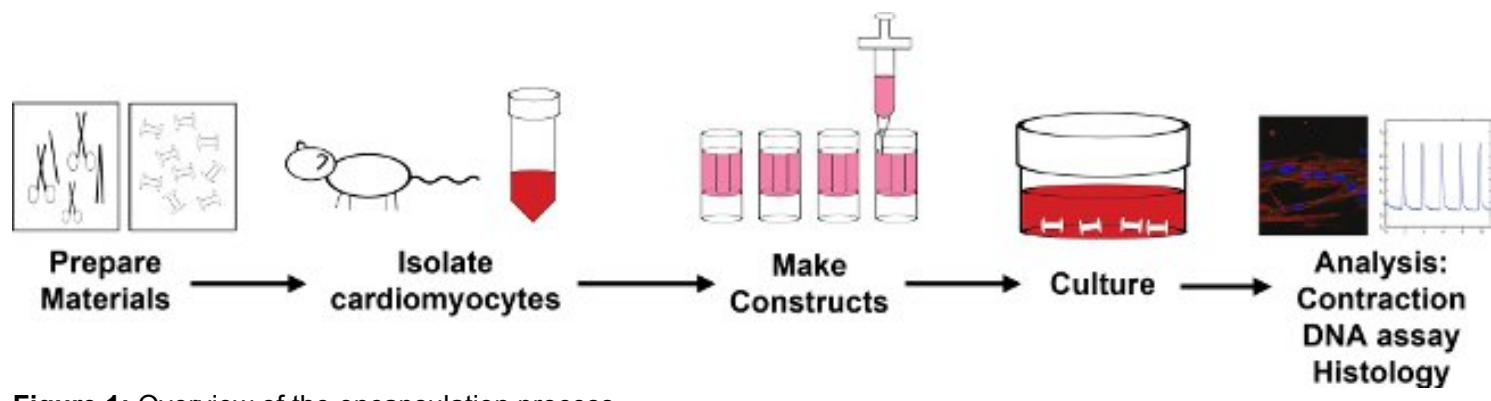

Figure 1: Overview of the encapsulation process

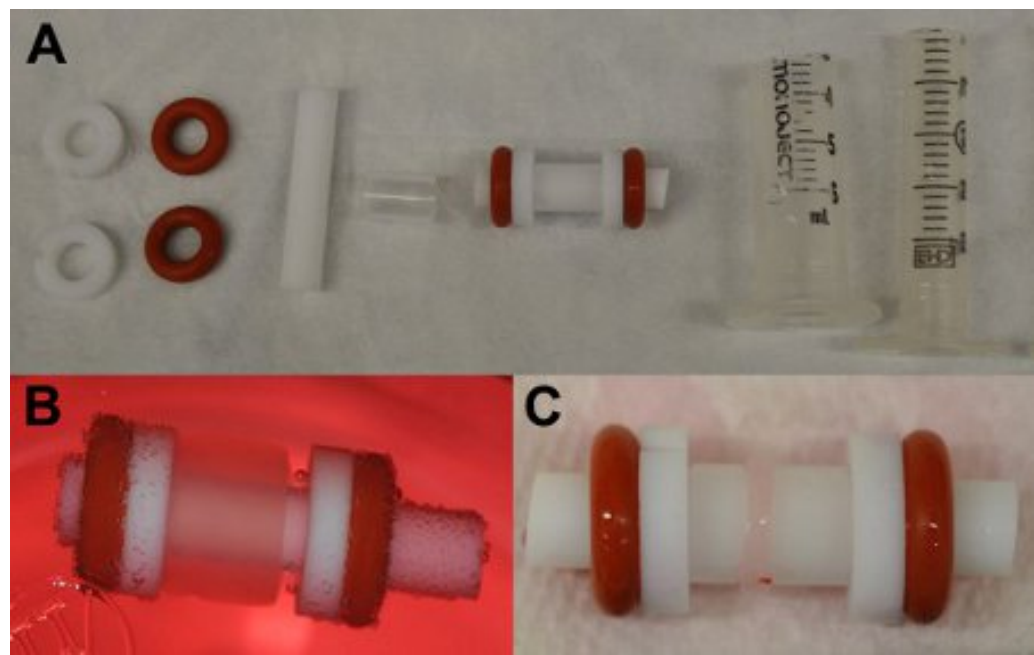

Figure 2: A) Separate and combined mold parts for creating fibrin gels. From left to right: two Teflon washers, two silicone o-rings, a Teflon rod, a Teflon tube, a completed mandrel, the outer casing for the mandrel, and the plunger). B) Construct on mold immediately after ejection from the outer casing (day 0). C) Compacted construct on mold (red arrow), following 13 days of culture.
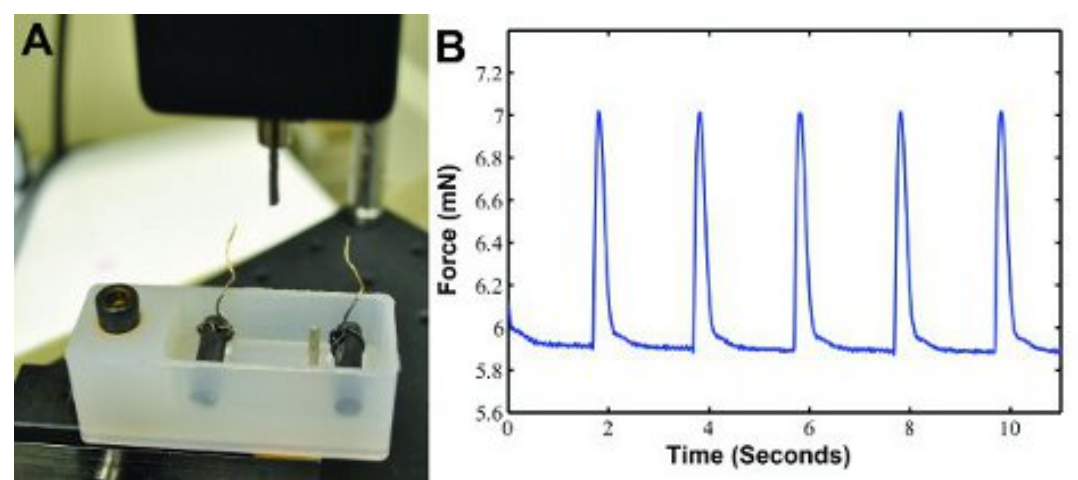

Figure 3: A) Custom contraction force measurement system for recording twitch force. A force transducer with a post measures the contraction force and outputs the results into a computer. A bath containing two carbon electrodes with wires connects to an electrical stimulator which paces the construct. The two posts hold the construct in place. B) Sample twitch force waveform data generated with electrical stimulation at 0.5 $\mathrm{Hz}$. 

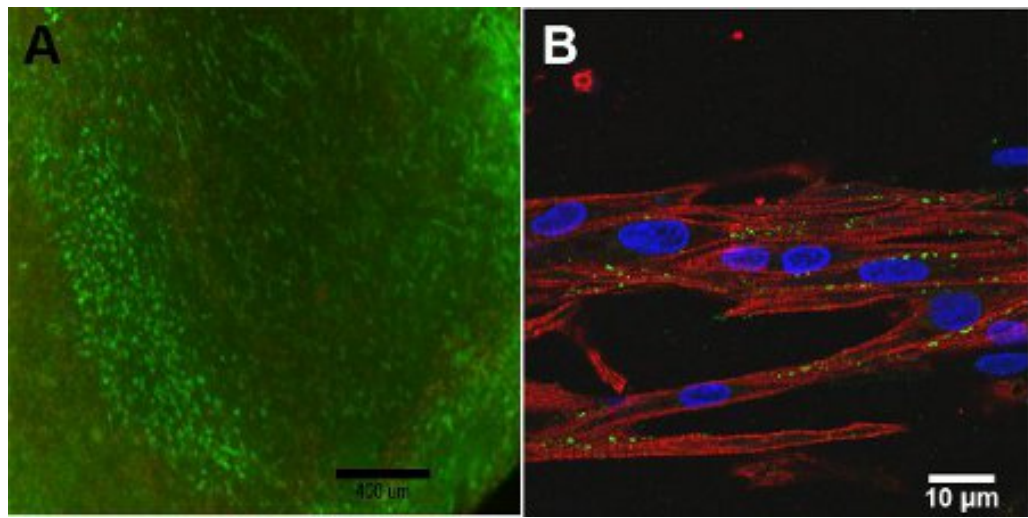

Figure 4: A) Live/Dead assay of construct, day 13 (scale bar $=400 \mu \mathrm{m})$. Green represents the live cells and red represents the dead cells. B) Confocal Image of Myosin Heavy Chain (red), Connexin 43 (green) and Hoescht nuclear stain (blue) (scale bar $=10 \mu \mathrm{m}$ ).

\begin{tabular}{|c|c|c|c|c|c|}
\hline \multicolumn{2}{|l|}{ F solution } & \multicolumn{2}{|l|}{ T solution } & \multicolumn{2}{|l|}{ Cell Solution } \\
\hline Fibrinogen & $112 \mu \mathrm{l}$ & Thrombin & $17 \mu \mathrm{l}$ & Cells in DMEM & $170 \mu \mathrm{l}$ \\
\hline \multirow[t]{2}{*}{ HEPES } & $558 \mu \mathrm{l}$ & $\mathrm{Ca}++$ & $1.3 \mu \mathrm{l}$ & & \\
\hline & & DMEM & $152 \mu \mathrm{l}$ & & \\
\hline Total & $670 \mu \mathrm{l}$ & Total & $170 \mu \mathrm{l}$ & Total & $170 \mu \mathrm{l}$ \\
\hline
\end{tabular}

Table 1. Fibrin gel solutions, quantities for $1 \mathrm{~mL}$ of gel.

Note: Fibrinogen $=33 \mathrm{mg} / \mathrm{mL}$ Fibrinogen in $20 \mathrm{mM}$ HEPES buffered Saline

HEPES $=20 \mathrm{mM}$ HEPES buffered saline

Thrombin $=25 \mathrm{U} / \mathrm{mL}$ solution in $0.81 \% \mathrm{NaCl}$ solution

$\mathrm{Ca}++=2 \mathrm{~N}$ Calcium Chloride solution

\section{Discussion}

The encapsulation of neonatal rat cardiomyocytes in fibrin gels results in a consistent and viable three-dimensional in vitro model of the myocardial system. Fibrin is a preferred biomaterial because when the cells are entrapped, they are metabolically active and capable of compacting, remodeling and recreating an extracellular matrix that is consistent with native heart tissue ${ }^{12}$. Because we allow the cardiomyocytes to align themselves in this environment, their functionality is more characteristic of cardiac muscle resulting in larger contraction force as compared to isotropic tissues 6 . For potential therapeutic applications, it is necessary to encapsulate cells within a material that promotes both viability and functionality. The protocols presented here demonstrate an efficient and accurate means for creating a fibrin network to control cardiac cell behavior in a three dimensional microenvironment.

A few potential issues may arise during the creation and culture of these constructs. One potential issue is the maintenance of cell viability prior to encapsulation, which will significantly affect the functionality of the construct. Effort should be made to limit cell death following the isolation by reducing the time between the isolation and the encapsulation of the cells within the fibrin gel. Constructs should be provided media every other day on a strict schedule. In addition, it is important to ensure homogeneity in all of the solutions used. If the mixture of fibrinogen, thrombin and cells produces a heterogeneous environment, the ability of the cells to remodel the ECM, mechanically couple and contract is potentially obstructed. It is also important to prevent the formation of air bubbles during the injection of the constructs in order to prevent disturbances in the continuity of the engineered tissue. One way to alleviate this issue is draw more gel than is needed to make the construct into the syringe and inject slowly. Lastly, once the fibrin matrix has set and has been in culture medium for 24 hours, it is essential to detach the construct from the sides of the ring mold to promote the cell-based compaction of the fibrin gel. Keeping the construct in the middle of the mold facilitates gas and nutrient exchange. Adherence to the sides of the ring mold may also disrupt the desired cellular alignment.

It is important to note that conscious decapitation is used as the method of euthanization in this protocol, which is an acceptable method under the guidelines from both the National Institutes of Health and the American Veterinary Medical Association. However, some institutions recommend anesthetic use followed by decapitation for neonatal rats. We have chosen conscious decapitation because it ensures the minimal amount of time under hypoxic conditions for the excised heart tissue/cells. Relatively small amounts of hypoxia can lead to myocyte ischemia and potentially myocyte death, which could significantly affect the outcomes of this protocol.

\section{Disclosures}

No conflicts of interest declared.

\section{Acknowledgements}

This work was supported by the National Institutes of Health - National Heart, Lung and Blood Institute (Award \# R00HL093358 to L.D.B.) 


\section{References}

1. Stegemann, J.P., Hong, H., \& Nerem, R.M. Mechanical, biochemical, and extracellular matrix effects on vascular smooth muscle cell phenotype. Journal of applied physiology. 98, 2321-7 (2005).

2. Guarnieri, D., et al. Covalently immobilized RGD gradient on PEG hydrogel scaffold influences cell migration parameters. Acta biomaterialia. 6, 2532-9 (2010).

3. Krebs, M.D., et al. Injectable poly(lactic-co-glycolic) acid scaffolds with in situ pore formation for tissue engineering. Acta biomaterialia. 5, 2847-59 (2009).

4. Zimmermann, W.-H. et al. Engineered heart tissue grafts improve systolic and diastolic function in infarcted rat hearts. Nature medicine. 12, 452-8 (2006).

5. Chung, C. \& Burdick, J.A. Influence of Three-Dimensional Hyaluronic Acid Stem Cell Chondrogenesis. Tissue engineering. 15 (Part A), (2009).

6. Black, L.D. et al. Cell-induced alignment augments twitch force in fibrin gel-based engineered myocardium via gap junction modification. Tissue engineering. 15 (Part A), 3099-108 (2009).

7. Syedain, Z.H., Weinberg, J.S., \& Tranquillo, R.T. Cyclic distension of fibrin-based tissue constructs: evidence of adaptation during growth of engineered connective tissue. Proceedings of the National Academy of Sciences of the United States of America. 105, 6537-42 (2008).

8. Falvo, M.R., Gorkun, O.V., \& Lord, S.T. The molecular origins of the mechanical properties of fibrin. Biophysical chemistry. 152, 15-20 (2010).

9. Jockenhoevel, S. et al. Fibrin gel - advantages of a new scaffold in cardiovascular tissue engineering. European journal of cardio-thoracic surgery : official journal of the European Association for Cardio-thoracic Surgery. 19, 424-30 (2001).

10. Ryan, E. Structural Origins of Fibrin Clot Rheology. Biophysical Journal. 77, 2813-2826 (1999).

11. Williams, C. et al. Cell sourcing and culture conditions for fibrin-based valve constructs. Tissue engineering. 12, 1489-502 (2006).

12. Grassl, E.D., Oegema, T.R. \& Tranquillo, R.T. A fibrin-based arterial media equivalent. Journal of biomedical materials research. 66 (Part A), 550-61 (2003).

13. Barocas, V.H. \& Tranquillo, R.T. An anisotropic biphasic theory of tissue-equivalent mechanics: the interplay among cell traction, fibrillar network deformation, fibril alignment, and cell contact guidance. Journal of biomechanical engineering. 119, 137-45(1997).

14. LIVE/DEAD Viability/Cytotoxicity Kit *for mammalian cells*. Small (2005). 